\title{
Letters
}

\section{Concepts and misconceptions regarding clinical staging models}

In an interesting editorial in the September issue, Alda and Kapczinski explore the clinical staging model as applied to bipolar disorder, ${ }^{1}$ consider some of the strengths and weaknesses of this approach and go on to comment on the predictions suggested by Scott and colleagues. ${ }^{2}$ Although the editorial rightly points out some of the limitations of current staging models, many of the authors' conclusions draw heavily on 2 ideas that are not core to most staging models, namely that staging is largely about established disorder and that disease progression is predominantly described by severity, with comorbidities treated as separate entities. Since these concepts have implications ranging from etiology to neurobiology, nosology and even clinical services, we believe that these additional issues need to be highlighted even though we agree with many of the sentiments and key messages in the editorial.

First, although the editorial's overall focus is on later stages of bipolar disorder, an important consideration for most of the staging models developed across mental health is the emphasis on early stages and subthreshold conditions - especially those presenting during the peak age of onset for severe mental disorders (15-25 yr). Unlike older adults with established suprathreshold disorders that meet traditional diagnostic criteria (where Alda and Kapczinski's distinction between "illness progression or typology of illness" may hold true), the early course of illness and even the long-term trajectories of many early-stage, subthreshold syndromes are pluripotential and much less predictable (e.g., some evolve into recurrent depression, bipolar disorder, or psychosis; others represent time-limited adjustment problems with no further episodes of any disorder). ${ }^{3,4}$ Staging models, applied in psychiatry as in general medicine, focus primarily on where an individual is located on a continuum - from an asymptomatic at-risk state through to end-stage disease thereby examining the full trajectory and course of illness from its early beginnings. Also in staging models, illness progression is not just about symptom severity; rather, it involves disease extension. In cancer, for example, the size of a primary tumour, its spread to other locations within the body and involvement of other systems are critical measures of stage in the tumour-node-metastasis model.

Second, staging models take a lifecourse perspective that transforms the impression of multiple lifetime psychiatric comorbidities (measured crosssectionally), recognizing that many of these may in fact be artifactual or transitory. In reality the picture is one of heterotypic continuities, with early, less specific stages of disorder potentially evolving into more specific diagnoses. This is commonly observed in general medicine, where obesity may be a precursor to diabetes, and obesity and hypertension may be comorbid with each other while also being precursors to heart disease. ${ }^{5,6}$ Similarly, bipolar-spectrum features (e.g., brief hypomania) may be a persistent disease pattern in older adults, yet when occurring in young people they may reflect a more dynamic state, representing an early stage of an illness trajectory; studies have demonstrated that about $20 \%$ of such cases progress to mania within 12-18 months. ${ }^{7}$

Furthermore, Alda and Kapczinski state that insufficient data exist to judge whether earlier treatments have better risk:benefit ratios than later ones. However, treatment in staging models dictates that interventions should be age- and stage-appropriate. ${ }^{8}$ This is especially pertinent for early subthreshold stages of illness, which may be self-limiting conditions or can revert to mild or nonspecific symptoms, especially with appropriate interventions. Because of this potential, we would neither anticipate nor recommend that first-line treatments for established bipolar disorder, such as lithium or anticonvulsants, be prescribed for subsyndromal, nonspecific conditions. ${ }^{9}$ As these represent the early stages of "potential bipolarity," the illness trajectory may still be unclear, and symptoms may overlap considerably with the early stages of other disorders. There is no evidence that these traditional treatments would be effective or appropriate. Overall, the risk:benefit ratio of psychosocial treatments is significantly lower than for disease-specific psychopharmacology, certainly in cases of emerging psychosis $^{10}$ and, as more recently suggested, in cases of stages 0-2 of bipolar disorder. ${ }^{11}$ This is why many services offer nonpharmacological interventions to the large numbers of help-seeking youth presenting with transdiagnostic early-stage disorders.

Alda and Kapczinski rightly point out that we cannot at this point demonstrate a change in the pattern of any mental disorder because of early intervention. However, in general medicine, the application of clinical staging models to the treatment of melanoma and breast cancer demonstrates unequivocally that early intervention does change the distribution and prognosis of disease. ${ }^{12}$ Of course, general medicine has the advantage that the pathophysiological boundaries between clinical stages can be measured with biomarkers rather than relying on clinical phenotypes. ${ }^{13}$

The new staging model of understanding clinical phenotypes represents a paradigm shift that has major implications for current research. It highlights that in order to identify biomarkers of illness onset and progression rather than treatment response, researchers must focus on early stages - especially at-risk syndromes (often designated as stage 0 and 1a) and subthreshold syndromes (stage 1b) as some of these will evolve to a full-threshold disorder meeting traditional diagnostic criteria (stage 2).${ }^{14}$ Perhaps most of all staging models pose a fundamental question for 
psychiatry, namely whether we need models for different disorders if their trajectories (especially early on) overlap in so many ways (e.g., genetics, neuropsychological profile, oxidative stress, sleep-wake cycle).${ }^{15}$ Pluripotential outcomes, transdiagnostic risk factors that could explain mental and physical comorbities, age at peak onset and transitions of subthreshold syndromes often defy classification under current schema, but are likely to be a key part of the new psychiatry. ${ }^{16}$ If we decide to adopt clinical staging models, whichever version we choose will challenge us all, as it is not just a case of putting old wine into new bottles (or young wine into old ones!).

Jai Shah, MD, MSc; Jan Scott, MB BS, PhD

Affiliations: From the PEPP-Montreal, Douglas Mental Health University Institute, ACCESS Open Minds, Department of Psychiatry, McGill University, Montreal, Que., Canada (Shah); and the Institute of Neuroscience, Newcastle University, \& Centre for Affective Disorders, IPPN, King's College, London, UK (Scott).

\section{DOI: 10.1503/jpn.160196}

\section{References}

1. Alda M, Kapczinski F. Staging model raises fundamental questions about the nature of bipolar disorder. J Psychiatry Neurosci 2016:41:291-3.

2. Scott J, Leboyer M, Hickie I, et al. Clinical staging in psychiatry: a cross-cutting model of diagnosis with heuristic and practical value. Br J Psychiatry 2013;202: 243-5.

3. Kim-Cohen J, Caspi A, Moffitt TE, et al. Prior juvenile diagnoses in adults with mental disorder: developmental followback of a prospective-longitudinal cohort. Arch Gen Psychiatry 2003;60:709-17.

4. Shankman SA, Lewinsohn PM, Klein DN, et al. Subthreshold conditions as precursors for full syndrome disorders: a 15-year longitudinal study of multiple diagnostic classes. J Child Psychol Psychiatry 2009;50:1485-94.

5. Wright JR. Albert C. Broders' paradigm shifts involving the prognostication and definition of cancer. Arch Pathol Lab Med 2012;136:1437-46.

6. Gonnella JS, Hornbrook MC, Louis DZ. Staging of disease. A case-mix measurement. JAMA 1984;251:637-44.

7. Bechdolf A, Ratheesh A, Cotton SM, et al. The predictive validity of bipolar atrisk (prodromal) criteria in help-seeking adolescents and young adults: a prospective study. Bipolar Disord 2014;16:493-504.
8. McGorry PD, Hickie IB, Yung AR, et al. Clinical staging of psychiatric disorders: a heuristic framework for choosing earlier safer and more effective interventions. Aust N Z J Psychiatry 2006;40:616-22.

9. Scott J, Hickie IB, McGorry P. Preemptive psychiatric treatments: Pipe dream or a realistic outcome of clinical staging models? Neuropsychiatry 2012;2: 263-6.

10. Liu P, Parker AG, Hetrick SE, et al. An evidence map of interventions across premorbid, ultra-high risk and first episode phases of psychosis. Schizophr Res 2010;123:37-44

11. Vallarino M, Henry C, Etain B, et al. An evidence map of psychosocial interventions for the earliest stages of bipolar disorder. Lancet Psychiatry 2015;2:548-63.

12. Lønning PE. Breast cancer prognostication and prediction: Are we making progress? Ann Oncol 2007;18(Suppl 8):viii3-7.

13. Tabák AG, Herder C, Rathmann W, et al. Prediabetes: a high-risk state for diabetes development. Lancet 2012;379: 2279-90.

14. McGorry P, Keshavan M, Goldstone S, et al. Biomarkers and clinical staging in psychiatry. World Psychiatry 2014;13: 211-23.

15. Bellivier F, Geoffroy PA, Scott J, et al. Biomarkers of bipolar disorder: Specific or shared with schizophrenia? Front Biosci (Elite Ed) 2013;5:845-63.

16. Insel TR. Translating scientific opportunity into public health impact: a strategic plan for research on mental illness. Arch Gen Psychiatry 2009;66:128-33.

\section{Author response}

We are thankful to Drs. Shah and Scott for their comments on our editorial. Their letter gives us an opportunity to expand on and clarify the most relevant points. Our main argument has been that the staging model may not be as fully developed as to be ready for clinical application, but it has had healthy stimulating effect on our field, and we see the letter by Shah and Scott as a testimony to that.

It was not our intention to focus just on stages in fully manifest illness. The importance of recognizing clinical heterogeneity of bipolar disorder is supported by studies of prodromal stages as well. We have been involved in some of the longitudinal high-risk studies of young people at risk for bipolar disorder. ${ }^{1-3}$ These studies, among others, described early stages of the illness and their comorbidities ${ }^{4}$ as well as neuropsychological functioning $^{5}$ and structural brain findings. ${ }^{6}$ Based on these studies as well as on observations of other authors, we believe that the point of heterogeneity of bipolar disorder and the need to separate it from the concept of uniformly progressing illness are equally applicable in the early stages as in the latter ones. The prodromes may appear nonspecific and uninformative with respect to future illness trajectories when viewed through the prism of current diagnostic classification. But an alternate phenotypic characterization may identify well circumscribed and more homogeneous subgroups of illness that are continuous with the later stages. This is exemplified by the studies of children of lithium-responsive and nonresponsive parents, showing concordance of the patterns of clinical course (as well as treatment response) between generations. ${ }^{4,7}$

Another point raised by Shah and Scott pertains to the risk:benefit ratio of treatments in different stages, considered more favourable early on. We agree that this is a reasonable assumption, but it remains to be tested, and such testing may not be easy to carry out. A recent review lists a handful of studies, some showing short-term relief of clinical symptoms in young people (frequently treated with medication). ${ }^{8}$ However, the lower risk:benefit ratio of these mostly psychosocial treatments is implicitly assumed rather than derived from appropriate comparisons.

We agree with Drs. Shah and Scott that more attention needs to be paid to the early stages of major psychiatric disorders. We also believe that the staging concept is important for psychiatry heuristically as it challenges some of the basic concepts of bipolar disorder. However, before it can be applied clinically, the staging model deserves deeper scrutiny and more support from longitudinal studies.

\section{Martin Alda, MD; Flavio Kapczinski, MD, PhD}

Affiliations: From the Department of Psychiatry, Dalhousie University, Halifax, NS, Canada (Alda); and the Department of Psychiatry and Behavioural Neurosciences, McMaster University, Hamilton, Ont., Canada (Kapczinski).

DOI: 10.1503/jpn.160197 


\section{References}

1. Duffy A, Alda M, Kutcher S, et al. Psychiatric symptoms and syndromes among adolescent children of parents with lithium-responsive or lithiumnonresponsive bipolar disorder. Am J Psychiatry 1998;155:431-3.

2. Duffy A, Alda M, Hajek T, et al. Early stages in the development of bipolar disorder. J Affect Disord 2010;121:127-35.

3. Uher R, Cumby J, MacKenzie LE, et al. A familial risk enriched cohort as a platform for testing early interventions to prevent severe mental illness. BMC Psychiatry 2014; 14:344.

4. Duffy A, Alda M, Kutcher S, et al. A prospective study of the offspring of bipolar parents responsive and nonresponsive to lithium treatment. J Clin Psychiatry 2002;63:1171-8.

5. MacQueen GM, Grof P, Alda M, et al. A pilot study of visual backward masking performance among affected versus unaffected offspring of parents with bipolar disorder. Bipolar Disord 2004;6:374-8.

6. Hajek T, Cullis J, Novak T, et al. Brain structural signature of familial predisposition for bipolar disorder: replicable evidence for involvement of the right inferior frontal gyrus. Biol Psychiatry 2013;73:144-52.

7. Duffy A, Alda M, Milin R, et al. A consecutive series of treated affected offspring of parents with bipolar disorder: Is response associated with the clinical profile? Can J Psychiatry 2007;52:369-76.

8. Vallarino M, Henry C, Etain B, et al. An evidence map of psychosocial interventions for the earliest stages of bipolar disorder. Lancet Psychiatry 2015;2:548-63. 